\title{
Robust Stabilization of Fractional-Order Systems with Interval Uncertainties via Fractional-Order Controllers
}

\section{Saleh Sayyad Delshad, Mohammad Mostafa Asheghan, and Mohammadtaghi Hamidi Beheshti}

\author{
Electrical Engineering Department, Tarbiat Modares University, P.O.Box 14115-349, Tehran, Iran \\ Correspondence should be addressed to \\ Mohammadtaghi Hamidi Beheshti, mbehesht@modares.ac.ir
}

Received 31 December 2009; Accepted 4 May 2010

Academic Editor: Josef Diblik

Copyright (C) 2010 Saleh Sayyad Delshad et al. This is an open access article distributed under the Creative Commons Attribution License, which permits unrestricted use, distribution, and reproduction in any medium, provided the original work is properly cited.

We propose a fractional-order controller to stabilize unstable fractional-order open-loop systems with interval uncertainty whereas one does not need to change the poles of the closed-loop system in the proposed method. For this, we will use the robust stability theory of Fractional-Order Linear Time Invariant (FO-LTI) systems. To determine the control parameters, one needs only a little knowledge about the plant and therefore, the proposed controller is a suitable choice in the control of interval nonlinear systems and especially in fractional-order chaotic systems. Finally numerical simulations are presented to show the effectiveness of the proposed controller.

\section{Introduction}

Recently, studying fractional-order differential systems has become an active research field. Even though fractional calculus is a mathematical topic with more than 300 years old history, its application to physics and engineering has attracted many researchers in different branches of control. It has been found that in interdisciplinary fields, many systems can be described by fractional differential equations [1-8]. These examples and similar researches perfectly clarify the importance of consideration and analysis of dynamical systems with fractional-order models. The $P I^{\lambda} D^{\mu}$ controller [9], the fractional lead-lag compensator [10], and the CRONE controllers are some of the famous FO controllers [11].

Stabilizing of FO systems (Linear or Nonlinear) with interval uncertainties is still open to our best knowledge. In [12], authors proposed a fractional-order controller to change the order of the overall closed-loop system to a desired fractional order when open-loop 
system was integer and has no interval uncertainty. For the first time, this paper will present a fractional-order controller to stabilize unstable fractional-order open-loop systems with interval uncertainty whereas one does not need to change the poles of the closed-loop system in the proposed method. Clearly, for closed-loop control systems, there are four situations. They are IO (integer order) plant with IO controller, IO plant with FO (fractional order) controller, FO plant with IO controller, and FO plant with FO controller. In this paper, we focus on using FO controllers for unstable FO systems and we propose a simple fractional-order controller to control of fractional-order interval systems. It is obvious that the considered formation covers IO plant.

The remaining part of this paper is organized as follows. Section 2 includes basic concepts in fractional calculus. In Section 3, we consider stability of the fractional-order linear and nonlinear systems. Using of two lemmas in Section 4, it is easy to calculate the lower and upper boundaries of interval eigenvalues separately in real part and imaginary part. Stability check via minimum argument of phase criteria is another discussion that is presented in this section. In order to achieve a robust stabilization of an FO-LTI, a fractional-order controller is proposed in Section 5 that can be extended to FO nonlinear systems and guarantees locally robust stability of considered system. Numerical simulation results are given in Section 6 to illustrate the effectiveness of the proposed controller. Finally, conclusions in Section 7 close the paper.

\section{Fractional Calculus}

\subsection{Definition}

The noninteger-order integro-differential operator, denoted by ${ }_{a} D_{t}^{\alpha}$, is a combined integration-differentiation operator commonly used in fractional calculus. This operator is defined by

$$
{ }_{a} D_{t}^{\alpha}= \begin{cases}\frac{d^{\alpha}}{d t^{\alpha}}, & \alpha>0 \\ 1, & \alpha=0, \\ \int_{a}^{t}(d \tau)^{-\alpha}, & \alpha<0 .\end{cases}
$$

There are some definitions for fractional derivatives [13]. The Riemann-Liouville definition is a common notation of fractional derivative. Accordingly, an $\alpha$ th order fractional derivative of function $f(t)$ with respect to time $t$ and the terminal value $a$ is given by

$$
{ }_{a} D_{t}^{\alpha} f(t)=\frac{d^{\alpha} f(t)}{d(t-a)^{\alpha}}=\frac{1}{\Gamma(n-\alpha)} \frac{d^{n}}{d t^{n}} \int_{0}^{t}(t-\tau)^{n-\alpha-1} f(\tau) d \tau
$$

where $n$ is the first integer which is not less than $\alpha$, that is, $n-1 \leq \alpha<n$ and $\Gamma$ is the Gamma function as

$$
\Gamma(z)=\int_{0}^{\infty} t^{z-1} e^{-t} d t
$$


The Laplace transforms of the Riemann-Liouville fractional integral and derivative are given as follows:

$$
\begin{gathered}
L\left\{{ }_{0} D_{t}^{\alpha} f(t)\right\}=s^{\alpha} F(s), \quad \alpha \leq 0, \\
L\left\{{ }_{0} D_{t}^{\alpha} f(t)\right\}=s^{\alpha} F(s)-\sum_{k=0}^{n-1} s^{k}{ }_{0} D_{t}^{\alpha-k-1} f(0), \quad n-1<\alpha \leq n \in N .
\end{gathered}
$$

For an initial problem of Riemann-Liouville type, one would have to specify the values of certain fractional derivatives of the unknown solution at the initial point $t=0$. However, it is not clear what the physical meanings of fractional derivatives of $x$ are when we are dealing with a concrete physical application, and hence it is also not clear how such quantities can be measured. The problem will be coped with the Caputo definition, which is sometimes called smooth fractional derivative. This is described by

$$
{ }_{0} D_{t}^{\alpha} f(t)= \begin{cases}\frac{1}{\Gamma(m-\alpha)} \int_{0}^{t} \frac{f^{(m)}(\tau)}{(t-\tau)^{\alpha+1-m}} d \tau, & m-1<\alpha<m \\ \frac{d^{m}}{d t^{m}} f(t), & \alpha=m\end{cases}
$$

where $m$ is the first integer larger than $\alpha$. It is found that the equations with RiemannLiouville operators are equivalent to those with Caputo operators by homogeneous initial conditions assumption [13]. The Laplace transform of the Caputo fractional derivative is

$$
L\left\{{ }_{0} D_{t}^{\alpha} f(t)\right\}=s^{\alpha} F(s)-\sum_{k=0}^{n-1} s^{\alpha-1-k} f^{(k)}(0), \quad n-1<\alpha \leq n \in N .
$$

According to (2.6), only integer-order derivatives of function $f$ appear in the Caputo fractional Laplace transformation. In the rest of this paper, the notation ${ }_{a} D_{t}^{\alpha}$ represents the Caputo fractional derivative.

\subsection{Approximation Methods}

The numerical calculation of a fractional differential equation is not simple as that of an ordinary differential equation. In the literatures of fractional chaos, two approximation methods have been proposed for numerical solution of a fractional differential equation. One is the frequency-domain method $[14,15]$ and another is the time-domain method that is based on the predictor-correctors scheme $[16,17]$. This method is an improved version of AdamsBashforth-Moulton algorithm [17-19].

Here we use a predictor-corrector algorithm for fractional-order differential equations. The brief introduction of this algorithm is as following.

Consider the following differential equation:

$$
D_{t}^{\alpha} x=f(t, x), \quad 0 \leq t \leq T, \quad x^{(k)}(0)=x_{0}^{(k)}, \quad k=0,1,2, \ldots, n-1
$$


which is equivalent to the Volterra integral equation [20]

$$
x=\sum_{k=0}^{[\alpha]-1} x_{0}^{(k)} \frac{t^{k}}{k !}+\frac{1}{\Gamma(\alpha)} \int_{0}^{t} \frac{f(\tau, x)}{(t-\tau)^{1-\alpha}} d \tau
$$

Set $h=T / N, t_{n}=n h(n=0,1,2, \ldots, N)$. Then (2.8) can be discretized as follows:

$$
x_{h}\left(t_{n+1}\right)=\sum_{k=0}^{[\alpha]-1} x_{0}^{(k)} \frac{t_{n+1}^{k}}{k !}+\frac{h^{\alpha}}{\Gamma(\alpha+2)} f\left(t_{n+1}, x_{h}^{p}\left(t_{n+1}\right)\right)+\frac{h^{\alpha}}{\Gamma(\alpha+2)} \sum a_{j, n+1} f\left(t_{j}, x_{h}\left(t_{j}\right)\right),
$$

where predicted value $x_{h}^{p}\left(t_{n+1}\right)$ is determined by

$$
\begin{gathered}
x_{h}^{p}\left(t_{n+1}\right)=\sum_{k=0}^{n-1} x_{0}^{(k)} \frac{t_{n+1}^{k}}{k !}+\frac{1}{\Gamma(\alpha)} \sum_{j=0}^{n} b_{j, n+1} f\left(t_{j}, x_{h}\left(t_{j}\right)\right), \\
a_{j, n+1}= \begin{cases}n^{\alpha+1}-(n-\alpha)(n+1)^{\alpha+1}, & j=0, \\
(n-j+2)^{\alpha+1}+(n-j)^{\alpha+1}-2(n-j+1)^{\alpha+1}, & 1 \leq j \leq n, \\
1, & j=n+1,\end{cases} \\
b_{j, n+1}=\frac{h^{\alpha}}{\alpha}\left((n-j+1)^{\alpha}-(n-j)^{\alpha}\right) .
\end{gathered}
$$

The estimation error in this method is calculated as

$$
e=\operatorname{Max}\left|x\left(t_{j}\right)-x_{h}\left(t_{j}\right)\right|=O\left(h^{p}\right) \quad(j=0,1, \ldots, N),
$$

in which $p=\operatorname{Min}(2,1+\alpha)$. By utilizing the above method, numerical solution of a fractionalorder equation with dimension $n$ can be determined.

Now, consider a 3D fractional-order system as below:

$$
\begin{aligned}
& D^{\alpha} x=f_{1}(x, y, z), \\
& D^{\alpha} y=f_{2}(x, y, z), \\
& D^{\alpha} z=f_{3}(x, y, z)
\end{aligned}
$$


for $0<\alpha \leq 1$ and initial condition $\left(x_{0}, y_{0}, z_{0}\right)$ system (2.12) can be discretized as follows:

$$
\begin{aligned}
& x_{n+1}=x_{0}+\frac{h^{\alpha}}{\Gamma(\alpha+2)}\left[f_{1}\left(x_{n+1}^{p}, y_{n+1}^{p}, z_{n+1}^{p}\right)+\sum_{j=0}^{n} \gamma_{1, j, n+1} f_{1}\left(x_{j}, y_{j}, z_{j}\right)\right], \\
& y_{n+1}=y_{0}+\frac{h^{\alpha}}{\Gamma(\alpha+2)}\left[f_{2}\left(x_{n+1}^{p}, y_{n+1}^{p}, z_{n+1}^{p}\right)+\sum_{j=0}^{n} \gamma_{2, j, n+1} f_{2}\left(x_{j}, y_{j}, z_{j}\right)\right], \\
& z_{n+1}=z_{0}+\frac{h^{\alpha}}{\Gamma(\alpha+2)}\left[f_{3}\left(x_{n+1}^{p}, y_{n+1}^{p}, z_{n+1}^{p}\right)+\sum_{j=0}^{n} \gamma_{3, j, n+1} f_{3}\left(x_{j}, y_{j}, z_{j}\right)\right],
\end{aligned}
$$

where

$$
\begin{gathered}
x_{n+1}^{p}=x_{0}+\frac{1}{\Gamma(\alpha)} \sum_{j=0}^{n} \omega_{1, j, n+1} f_{1}\left(x_{j}, y_{j}, z_{j}\right), \\
y_{n+1}^{p}=y_{0}+\frac{1}{\Gamma(\alpha)} \sum_{j=0}^{n} \omega_{2, j, n+1} f_{2}\left(x_{j}, y_{j}, z_{j}\right), \\
z_{n+1}^{p}=z_{0}+\frac{1}{\Gamma(\alpha)} \sum_{j=0}^{n} \omega_{3, j, n+1} f_{3}\left(x_{j}, y_{j}, z_{j}\right), \\
r_{i, j, n+1}= \begin{cases}n^{\alpha+1}-(n-\alpha)(n+1)^{\alpha+1}, & j=0, \\
(n-j+2)^{\alpha+1}+(n-j)^{\alpha+1}-2(n-j+1)^{\alpha+1}, & 1 \leq j \leq n, \\
1, & j=n+1, \\
\omega_{i, j, n+1}=\frac{h^{\alpha}}{\alpha}\left((n-j+1)^{\alpha}-(n-j)^{\alpha}\right), \quad 0 \leq j \leq n, & i=1,2,3 .\end{cases}
\end{gathered}
$$

In the simulations of this paper, we use the above method to solve the fractional-order differential equations.

\section{Stability of FO-LTI System}

We consider the FO-LTI system with interval uncertainties in the parameters as follows:

$$
D_{t}^{\alpha} x(t)=A x(t)
$$

in which $\alpha$ is a noninteger number and $A \in A^{I}=[\underline{A}, \bar{A}]$.

With no interval uncertainty, it is well known that the stability condition of an FO-LTI system $D_{t}^{\alpha} x(t)=A x(t)$ is as in the following lemma. 
Lemma 3.1 (see [21]). The following autonomous system:

$$
D_{t}^{\alpha} x(t)=A x(t)
$$

with $0<\alpha<1, x \in R^{n}$, and $A \in R^{n \times n}$, is asymptotically stable if and only if $|\arg (\lambda)|>\alpha \pi / 2$ is satisfied for all eigenvalues $(\lambda)$ of matrix $A$. Also, this system is stable if and only if $|\arg (\lambda)| \geq \alpha \pi / 2$ is satisfied for all eigenvalues $(\lambda)$ of matrix $A$ with those critical eigenvalues satisfying $|\arg (\lambda)|=\alpha \pi / 2$ having geometric multiplicity of one. The geometric multiplicity of an eigenvalue $\lambda$ of the matrix $A$ is the dimension of the subspace of vectors $v$ for which $A v=\lambda v$.

if

Then, our robust stability test task for FO-LTI interval systems amounts to examining

$$
\min _{i}\left|\arg \left(\lambda_{i}(A)\right)\right|>\frac{\alpha \pi}{2}, \quad i=1,2, \ldots, N, \forall A \in A^{I}
$$

Consider the following nonlinear commensurate fractional-order system:

$$
D^{\alpha} x=f(x),
$$

where $0<\alpha<1$ and $x \in R^{n}$. The fixed points of system (3.4) are calculated by solving equation $f(x)=0$. These points are locally asymptotically stable if all eigenvalues of the Jacobian matrix $J=\partial f / \partial x$ evaluated at the fixed points satisfy [22]

$$
\min _{i}\left|\arg \left(\lambda_{i}(J)\right)\right|>\frac{\pi}{2}, \quad i=1,2, \ldots, N,
$$

where $\lambda_{i}$ is $i$ th the eigenvalue of matrix $J$. Here, we focus on the uncertain fractional-order nonlinear systems with interval Jacobian matrix.

\section{Robust Stability of FO-LTI Interval System}

From previous section, for the robust stability check of the uncertain fractional system, it is required to calculate the arguments of phase of eigenvalues. When there is no model uncertainty, it is easy to find the argument of phase of each eigenvalue. That is, by simply calculating

$$
\phi_{i}=\left|\arctan \left(\frac{\xi_{i}}{\sigma_{i}}\right)\right|, \quad i=1, \ldots, N
$$

where $N$ is number of eigenvalues of $A, \sigma_{i}=\operatorname{Re}\left\{\lambda_{i}\right\}$ and $\xi_{i}=\operatorname{Im}\left\{\lambda_{i}\right\}$ of eigenvalue $\lambda_{i}$, and finding the minimum $\phi_{i}$ such as

$$
\phi^{*}=\inf \left\{\phi_{1}, \ldots, \phi_{N}\right\}
$$

If $\phi^{*}>\alpha \pi / 2$, then the fractional system is considered stable. However, when there is model uncertainty, it is not easy to find (4.2) because $\lambda_{i}$ is not a fixed point in complex plane, instead 
it is a cluster of infinite points so that boundaries of considered cluster are calculated via the below subsection.

\subsection{Boundaries of Eigenvalues}

To identify eigenvalues of uncertain fractional-order system, the following interval matrix is defined:

$$
A^{I}=\left[A^{c}-\Delta A, A^{c}+\Delta A\right]
$$

where $A^{c}$ is a center matrix that is defined as nominal plant without uncertainty, and $\Delta A$ is a radius matrix corresponding to interval uncertainty.

Lemma 4.1 (see [23]). Define a sign calculation operator evaluated at $A^{c}$ such as

$$
P^{i}:=\operatorname{sign}\left[\left(u_{i}^{r e} v_{i}^{r e}-u_{i}^{i m} v_{i}^{i m}\right)^{T}\right]
$$

where $v_{i}$ and $u_{i}$ are left and right eigenvectors corresponding to ith eigenvalue of center matrix $A^{c}$, respectively, and $u_{i}^{r e}, v_{i}^{r e}, u_{i}^{i m}$, and $v_{i}^{i m}$ are defined as

$$
\begin{array}{ll}
u_{i}^{r e}=\operatorname{Re}\left(u_{i}\right), & u_{i}^{i m}=\operatorname{Im}\left(u_{i}\right), \\
v_{i}^{r e}=\operatorname{Re}\left(v_{i}\right), & v_{i}^{i m}=\operatorname{Im}\left(v_{i}\right) .
\end{array}
$$

If $P^{i}$ is constant for all $A^{I}$, then the lower and upper boundaries of the real part of ith interval eigenvalue are calculated as

$$
\underline{l}_{i}^{r e}=O_{i}^{r e}\left(A^{c}-\Delta A \circ P^{i}\right)
$$

where $O_{i}^{r e}(\cdot)$ is an operator for selecting the ith real eigenvalue of $(\cdot)$ and $C=A \circ B$ are $c_{k j}=a_{k j} b_{k j}$, and

$$
\bar{\lambda}_{i}^{r e}=O_{i}^{r e}\left(A^{c}+\Delta A \circ P^{i}\right)
$$

Lemma 4.2 (see [23]). Define a sign calculation operator evaluated at $A^{c}$ such as

$$
Q^{i}:=\operatorname{sign}\left[\left(u_{i}^{r e} v_{i}^{r e}+u_{i}^{i m} v_{i}^{i m}\right)^{T}\right]
$$

If $Q^{i}$ is constant for all $A^{I}$, then the lower and upper boundaries of the imaginary part of ith interval eigenvalue are calculated as

$$
\underline{\lambda}_{i}^{i m}=O_{i}^{i m}\left(A^{c}-\Delta A \circ Q^{i}\right)
$$


where $O_{i}^{i m}(\cdot)$ is an operator for selecting the ith imaginary eigenvalue, and

$$
\bar{\lambda}_{i}^{i m}=O_{i}^{i m}\left(A^{c}+\Delta A \circ Q^{i}\right)
$$

Thus, by utilizing Lemmas 4.1 and 4.2, the lower and upper boundaries of interval eigenvalue separately in real part and imaginary part are calculated. From above Lemmas, first $P^{i}$ and $Q^{i}, i=1, \ldots, N$ are calculated, then, interval ranges of eigenvalues are finally calculated as

$$
\lambda_{i}^{I}:=\left\{\left[\underline{\lambda}_{i}^{\mathrm{re}}, \bar{\lambda}_{i}^{\mathrm{re}}\right]+j\left[\underline{\lambda}_{i}^{\mathrm{im}}, \bar{\lambda}_{i}^{\mathrm{im}}\right]\right\}
$$

where $j$ represents imaginary part.

\subsection{Robust Stability Check}

From (4.2), since the stability condition is given as $\phi^{*}>\alpha \pi / 2$, if we find sufficient condition for this, the stability can be checked. For calculating $\phi^{*}$, the following procedure can be used [23].

(P1) Calculate $P_{i}$ and $Q_{i}$ for $i=1, \ldots, N$.

(P2) Calculate $\underline{\lambda}_{i}^{\mathrm{re}}, \bar{\lambda}_{i}^{\mathrm{re}}, \underline{\lambda}_{i}^{\mathrm{im}}$, and $\bar{\lambda}_{i}^{\mathrm{im}}$ for all $i \in\{1,2, \ldots, N\}$.

(P3) Find arguments of phase of four points such as

$$
\begin{array}{ll}
\phi_{i}^{1}=\angle\left(\underline{\lambda}_{i}^{\mathrm{re}}, \underline{\lambda}_{i}^{\mathrm{im}}\right), & \phi_{i}^{2}=\angle\left(\underline{\lambda}_{i}^{\mathrm{re}}, \bar{\lambda}_{i}^{\mathrm{im}}\right), \\
\phi_{i}^{3}=\angle\left(\bar{\lambda}_{i}^{\mathrm{re}}, \bar{\lambda}_{i}^{\mathrm{im}}\right), & \phi_{i}^{4}=\angle\left(\bar{\lambda}_{i}^{\mathrm{re}}, \underline{\lambda}_{i}^{\mathrm{im}}\right),
\end{array}
$$

in the complex plane.

(P4) Find $\phi_{i}^{*}=\inf \left\{\left|\phi_{i}^{1}\right|,\left|\phi_{i}^{2}\right|,\left|\phi_{i}^{3}\right|,\left|\phi_{i}^{4}\right|\right\}$.

(P5) Repeat procedures (P3) and (P4) for $i=1, \ldots, N$.

(P6) Find $\phi^{*}=\inf \left\{\phi_{i}^{*}, i=1, \ldots, N\right\}$.

(P7) If $\phi^{*}>\alpha \pi / 2$, then the fractional interval system is robust stable. Otherwise, the robust stability of system cannot be guaranteed.

\section{Controller Design}

A well-designed control system will have desirable performance. Moreover, a well-designed control system will be tolerant of imperfections in the model or changes that occur in the system. This important quality of a control system is called robustness [24]. It is obvious that open-loop and closed-loop systems with the same poles can exhibit different stability property if stability regions for these systems are different. Different stability regions are 
obtained when the open-loop and closed-loop systems have different orders. For this reason, a controller in order to change the order of the closed-loop system to a specific fractional order is designed. In [12], authors proposed a fractional-order controller to change the order of the overall closed-loop system to a desired fractional order when open-loop system was integer and has no interval uncertainty. To the best of our knowledge, stabilization of fractional-order open-loop systems with interval uncertainties via fractional-order controllers has not been considered yet. Therefore, in this paper we propose a fractional-order controller to stabilize unstable and uncertain fractional order open-loop systems whereas one does not need to change the poles of the closed-loop system in the proposed method. First, we assume that the uncertain system is described by an interval linear model given as follows:

$$
D^{\alpha} x(t)=\left(A^{c}+\Delta A\right) x(t)+u(t)
$$

where $x \in R^{n}, u \in R^{n}, A^{c}$ is an $n \times n$ center matrix, and $\Delta A$ is an $n \times n$ radius matrix corresponding to interval uncertainty. Assume that the control objective is to stabilize the closed-loop system. To achieve the goal, the below theorem is considered.

Theorem 5.1. Based on the control law $(t)=D^{\alpha} x(t)-D^{\alpha_{\text {controller }}} x(t)$, fractional interval system (5.1) will be robust stable if

$$
\alpha_{\text {controller }}<\frac{2 \phi^{*}}{\pi}
$$

where $\alpha_{\text {controller }}$ is order of controller and $\phi^{*}=\inf \left\{\phi_{1}, \ldots, \phi_{N}\right\}$.

Proof. Due to applying the control law $u$ as follows:

$$
u(t)=D^{\alpha} x(t)-D^{\alpha_{\text {controller }}} x(t),
$$

the closed-loop system is described as below:

$$
D^{\alpha_{\text {controller }}} x(t)=\left(A^{c}+\Delta A\right) x(t) \text {. }
$$

Now, using Lemmas 4.1 and 4.2 in Section 4 , and selection of $\alpha_{\text {controller }}$ as the following:

$$
\alpha_{\text {controller }}<\frac{2 \phi^{*}}{\pi}
$$

fractional interval system (5.1) will be robust stable.

For an uncertain plant with nonlinear fractional-order dynamics, we have

$$
D^{\alpha} x(t)=f_{\Delta}(x)+u(t),
$$


where $f_{\Delta}(x)$ is uncertain with interval uncertainty in the parameters. By calculating the Jacobian matrix of nonlinear system (5.6) at fixed points, we have

$$
J(x)=\left.\frac{\partial f}{\partial x}\right|_{x *}=J^{c}+\Delta J
$$

where $J^{c}$ is an $n \times n$ center matrix and $\Delta J$ is an $n \times n$ radius matrix corresponding to interval uncertainty. From Section 4 , it is evident that by applying the proposed controller in Theorem 5.1, the closed-loop dynamics will be locally robust stable if the parameter $\boldsymbol{\alpha}_{\text {controller }}$ is properly selected.

\section{Simulation Results}

\subsection{Stabilizing an Unstable FO-LTI Interval System}

Consider the following system [23]:

$$
D^{\alpha} x(t)=\left(A^{c}+\Delta A\right) x(t)+u(t)
$$

where,

$$
A^{c}=\left[\begin{array}{ccc}
-1 & -0.5 & -2 \\
1 & 0.5 & -0.5 \\
-0.5 & 2.5 & 1.2
\end{array}\right], \quad \Delta A=\left[\begin{array}{ccc}
0.1 & 0.05 & 0.2 \\
0.1 & 0.05 & 0.05 \\
0.05 & 0.25 & 0.12
\end{array}\right]
$$

and $\alpha=0.9$. Eigenvalues of center matrix $A^{c}$ are calculated as $\lambda_{1}=-1.7486, \lambda_{2}=1.2243+$ $j 1.5597$, and $\lambda_{3}=1.2243-j 1.5597$. It is obvious that the system is unstable. To robust stabilize the system via the proposed controller, $\alpha_{\text {controller }}$ as the control parameter should be chosen to satisfy condition (5.2) for $\lambda_{2,3}$. Now, from procedures (P1)-(P6), we find $\phi^{*}=0.7836$. So, from (5.2), we select $\alpha_{\text {controller }}<2 \phi^{*} / \pi=0.4989$ and conclude that the fractional interval system (6.1) is robust stable.

The numerical simulation has carried out using MATLAB subroutines written based on the method described in Section 2. The time step size employed in the simulation is 0.01 $(h=0.01)$. The simulation results are given in Figures 1 and 2 , when the controller has started to work at time $t=10$ seconds. In this example, the control parameter has been chosen as $\alpha=0.4$. As one can see, the maximum control efforts in this example are $5 \times 10^{6}, 4.9 \times 10^{6}$, and $0.6 \times 10^{7}$.

\subsection{Chaos Control of Fractional-Order Interval Arneodo System via Proposed Controller}

In dynamical systems, a saddle point is called a fixed point that has at least one eigenvalue in stable region and one eigenvalue in unstable region. In a three-dimensional system, if one of the eigenvalues is unstable and other eigenvalues are stable, then the equilibrium point is called saddle point of index 1 . By similar definition, a saddle point of index 2 is a saddle 

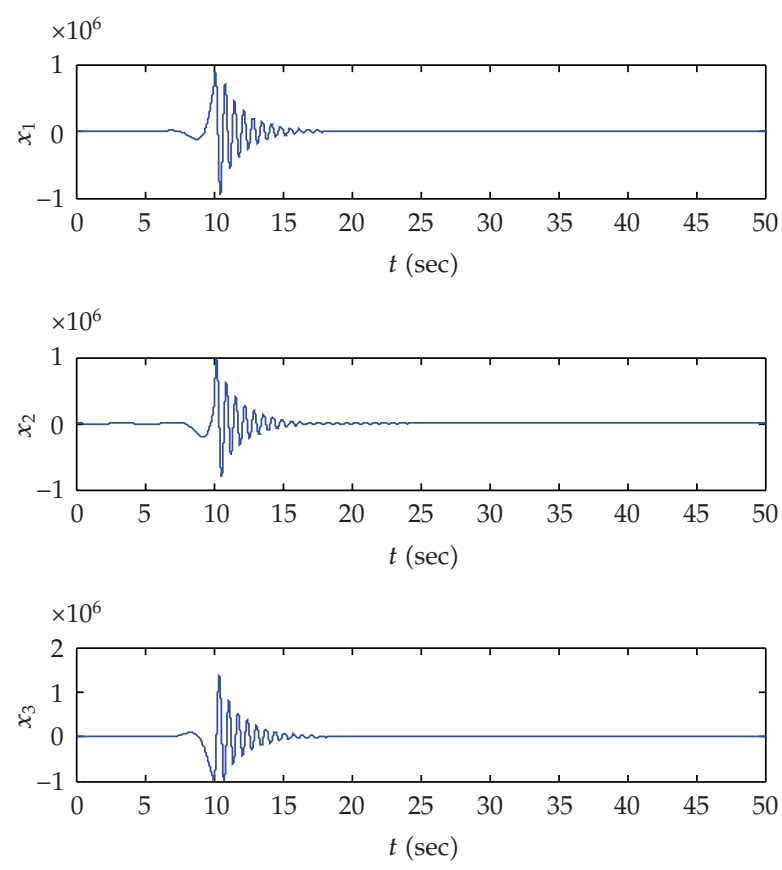

Figure 1: The time response of the states.
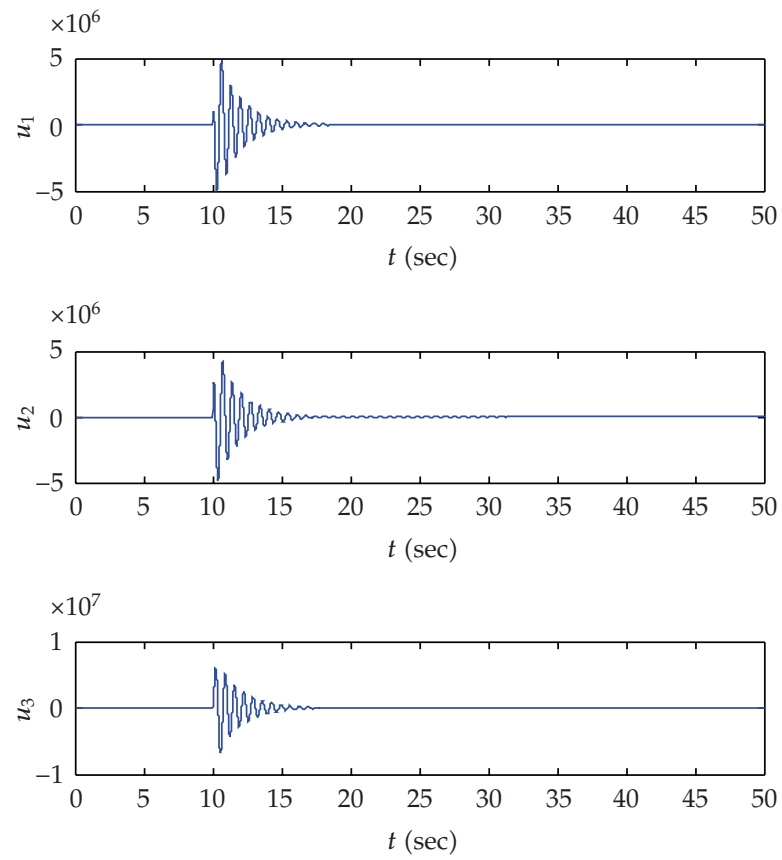

Figure 2: Control efforts. 
point with one stable eigenvalue and two unstable eigenvalues. In a chaotic system, scrolls are generated only around the saddle points of index 2 .

To control a three-dimensional chaotic system, saddle points of index 2 should be stabilized. By utilizing control law as (5.2), this goal is accessible by changing stable region via the proposed controller (5.3). Here, order of the overall closed-loop system is changed without any variation in eigenvalues of the fixed points.

Now, we consider the fractional-order Arneodo's system [25] as following:

$$
\begin{gathered}
D^{\alpha} x_{1}(t)=x_{2}(t), \\
D^{\alpha} x_{2}(t)=x_{3}(t), \\
D^{\alpha} x_{3}(t)=-\beta_{1} x_{1}(t)-\beta_{2} x_{2}(t)-\beta_{3} x_{3}(t)+\beta_{4} x_{1}^{3},
\end{gathered}
$$

where $\beta_{1}, \beta_{2}, \beta_{3}$, and $\beta_{4}$ are constant parameters. Nominal model of this system is found to be chaotic for the parameters $\beta_{1}=-5.5, \beta_{2}=3.5, \beta_{3}=1$, and $\beta_{4}=-1$.

Here, we assume that the mentioned parameters have interval uncertainty. Their corresponding uncertainties are as following:

$$
\begin{gathered}
\beta_{1}^{c}=-5.5, \quad \Delta \beta_{1}=0.1, \\
\beta_{2}^{c}=3.5, \quad \Delta \beta_{2}=0.1, \\
\beta_{3}^{c}=1, \quad \Delta \beta_{3}=0.1, \\
\beta_{4}^{c}=-1, \quad \Delta \beta_{4}=0.1 .
\end{gathered}
$$

With the given interval uncertainties, the fractional-order Arneodo's system has three fixed points as following:

$$
x_{1, \mathrm{eq}}=(0,0,0), \quad x_{2, \mathrm{eq}}=\left(\beta_{1}^{c}+\Delta \beta_{1}, 0,0\right), \quad x_{3, \mathrm{eq}}=\left(-\left(\beta_{1}^{c}+\Delta \beta_{1}\right), 0,0\right) .
$$

The Jacobian matrix of system (6.3), evaluated at $\left(x_{1, \mathrm{eq}}, x_{2, \mathrm{eq}}, x_{3, \mathrm{eq}}\right)$, is

$$
J=\left[\begin{array}{ccc}
0 & 1 & 0 \\
0 & 0 & 1 \\
-\left(\beta_{1}^{c}+\Delta \beta_{1}\right)+3\left(\beta_{4}^{c}+\Delta \beta_{4}\right) x_{1, \mathrm{eq}}^{2} & -\left(\beta_{2}^{c}+\Delta \beta_{2}\right) & -\left(\beta_{3}^{c}+\Delta \beta_{3}\right)
\end{array}\right]
$$

or

$$
J^{c}=\left[\begin{array}{ccc}
0 & 1 & 0 \\
0 & 0 & 1 \\
-\beta_{1}^{c}+3 \beta_{4}^{c} x_{1, \mathrm{eq}}^{2} & -\beta_{2}^{c} & -\beta_{3}^{c}
\end{array}\right],
$$



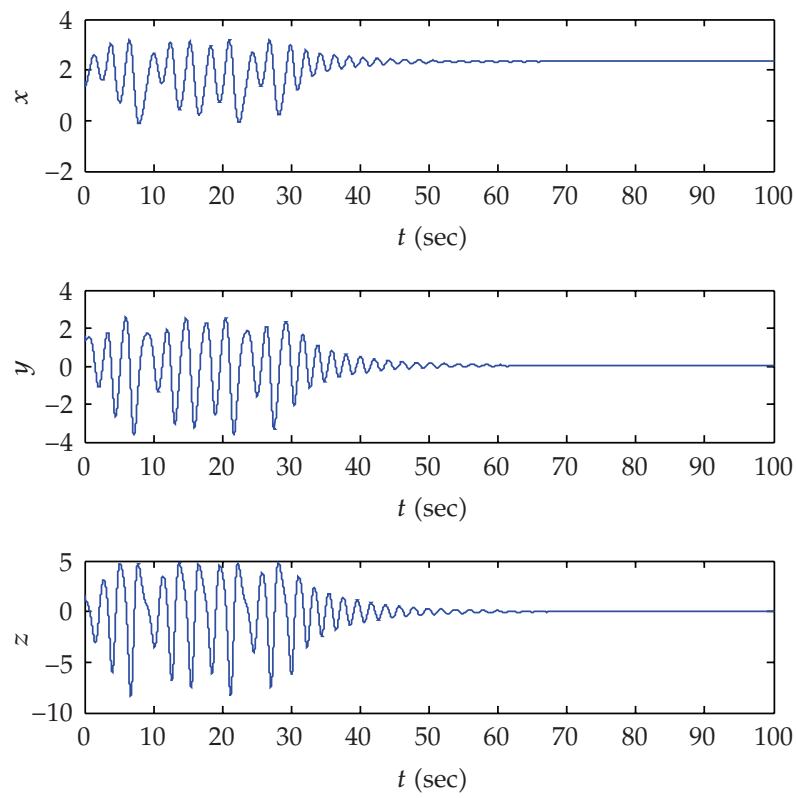

Figure 3: The time response of the states (stabilizing $x_{2, \mathrm{eq}}, x_{3, \mathrm{eq}}$ ).

$$
\Delta J=\left[\begin{array}{ccc}
0 & 1 & 0 \\
0 & 0 & 1 \\
-\Delta \beta_{1}+3 \Delta \beta_{4} x_{1, \mathrm{eq}}^{2} & -\Delta \beta_{2} & -\Delta \beta_{3}
\end{array}\right] .
$$

The fixed points and their corresponding eigenvalues for $J^{c}$ are calculated as follows:

$$
\begin{gathered}
x_{1, \mathrm{eq}}=(0,0,0): \lambda_{1}=1, \quad \lambda_{2,3}=-1 \pm j 2.1213, \\
x_{2, \mathrm{eq}}=(5.5,0,0): \lambda_{1}=-2, \quad \lambda_{2,3}=0.5 \pm j 2.2913, \\
x_{3, \mathrm{eq}}=(-5.5,0,0): \lambda_{1}=-2, \quad \lambda_{2,3}=0.5 \pm j 2.2913 .
\end{gathered}
$$

Necessary conditions to check the existence of chaos in fractional systems with commensurate or incommensurate rational orders are given in [26, 27], respectively. Based on [26], a necessary condition for fractional systems (6.3) to be chaotic is

$$
q>\max _{i}\left(\frac{2}{\pi} \tan ^{-1}\left(\frac{2.2913}{0.5}\right)\right)=0.8632 .
$$

According to (6.9), fixed points $x_{2, \text { eq }}$ and $x_{3, \text { eq }}$ are saddle points of index 2. From procedures (P1)-(P6), and then by using Theorem 5.1, when control parameter $\alpha_{\text {controller }}$ is chosen less than 0.8509 , eigenvalues of the fixed points $x_{2, \text { eq }}$ and $x_{3, \text { eq }}$ settle in the robust stable region and closed-loop system will be locally robust stable. Figures 3 and 4 exhibit simulation results when the controller with $\alpha_{\text {controller }}=0.8$ has been applied at time $t=30$ seconds.

Here, the maximum control efforts in this example are $0.6534,0.9771$, and 3.3212 . 

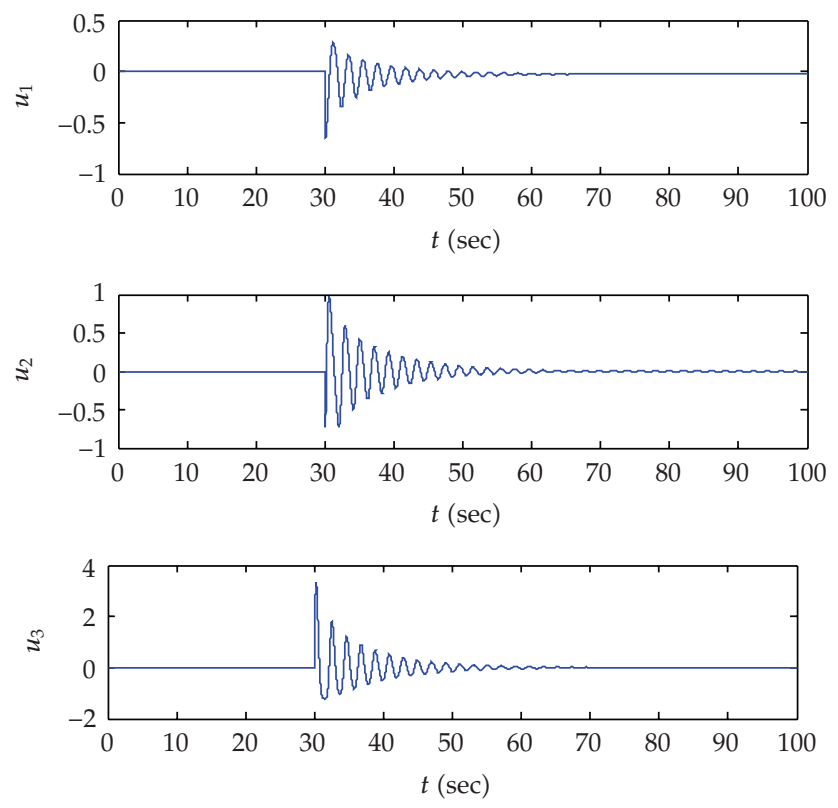

Figure 4: Control efforts.

\section{Conclusion}

In this paper, based on the robust stability theory of FO-LTI systems, we proposed a fractional-order controller to stabilize the unstable fixed points of an unstable open-loop fractional-order interval system. To determine the control parameters, one needed only a little knowledge about the plant and therefore, the proposed controller was a terrific choice in the control of fractional-order interval nonlinear systems. Finally, numerical simulations are also provided to show effectiveness of proposed controller in order to achieve robust stabilization of unstable and interval fractional-order systems.

\section{References}

[1] R. L. Bagley and R. A. Calico, "Fractional order state equations for the control of visco-elastically damped structures," Journal of Guidance, Control, and Dynamics, vol. 14, pp. 304-311, 1991.

[2] N. Engheta, "On fractional calculus and fractional multipoles in electromagnetism," IEEE on Transactions on Antennas and Propagation, vol. 44, no. 4, pp. 554-566, 1996.

[3] H. Linares, Ch. Baillot, A. Oustaloup, and Ch. Ceyral, "Generation of a fractal ground: application in robotics," in Proceedings of the IMACS-IEEE Multiconference on Computational Engineering in Systems Applications (CESA '96), Lille, France, July 1996.

[4] H.-F. Raynaud and A. Zergaïnoh, "State-space representation for fractional order controllers," Automatica, vol. 36, no. 7, pp. 1017-1021, 2000.

[5] A. Oustaloup, "Fractional order sinusoidal oscilators: optimization and their use in highly linear FM modulators," IEEE Transactions on Circuits and Systems, vol. 28, no. 10, pp. 1007-1009, 1981.

[6] M. Axtell and M. E. Bise, "Fractional calculus applications in control systems," in Proceedings of the IEEE of the National Aerospace and Electronics Conference, vol. 2, pp. 563-566, New York, NY, USA, 1990.

[7] J. A. T. Machado, "Special issue on fractional calculus and applications," Nonlinear Dynamics, vol. 29, pp. 1-386, 2002. 
[8] D. Xue, C. Zhao, and Y. Chen, "Fractional order PID control of A DC-motor with elastic shaft: a case study," in Proceedings of the IEEE American Control Conference (ACC '06), pp. 3182-3187, Minneapolis, Minn, USA, June 2006.

[9] I. Podlubny, "Fractional-order systems and $P I^{\lambda} D^{\mu}$-controllers," IEEE Transactions on Automatic Control, vol. 44, no. 1, pp. 208-214, 1999.

[10] H.-F. Raynaud and A. Zergaïnoh, "State-space representation for fractional order controllers," Automatica, vol. 36, no. 7, pp. 1017-1021, 2000.

[11] A. Oustaloup, J. Sabatier, and P. Lanusse, "From fractal robustness to the CRONE control," Fractional Calculus \& Applied Analysis, vol. 2, no. 1, pp. 1-30, 1999.

[12] M. S. Tavazoei and M. Haeri, "Chaos control via a simple fractional-order controller," Physics Letters A, vol. 372, no. 6, pp. 798-807, 2008.

[13] I. Podlubny, Fractional Differential Equations, vol. 198 of Mathematics in Science and Engineering, Academic Press, San Diego, Calif, USA, 1999.

[14] A. Charef, H. H. Sun, Y.-Y. Tsao, and B. Onaral, "Fractal system as represented by singularity function," IEEE Transactions on Automatic Control, vol. 37, no. 9, pp. 1465-1470, 1992.

[15] A. Charef, "Analogue realisation of fractional-order integrator, differentiator and fractional $P I^{\lambda} D^{\mu}$ controller," IEE Proceedings: Control Theory and Applications, vol. 153, no. 6, pp. 714-720, 2006.

[16] K. Diethelm, "An algorithm for the numerical solution of differential equations of fractional order," Electronic Transactions on Numerical Analysis, vol. 5, pp. 1-6, 1997.

[17] K. Diethelm, N. J. Ford, and A. D. Freed, "A predictor-corrector approach for the numerical solution of fractional differential equations," Nonlinear Dynamics, vol. 29, no. 1-4, pp. 3-22, 2002.

[18] K. Diethelm, N. J. Ford, and A. D. Freed, "Detailed error analysis for a fractional Adams method," Numerical Algorithms, vol. 36, no. 1, pp. 31-52, 2004.

[19] C. Li and G. Peng, "Chaos in Chen's system with a fractional order," Chaos, Solitons and Fractals, vol. 22, no. 2, pp. 443-450, 2004.

[20] K. Diethelm and N. J. Ford, "Analysis of fractional differential equations," Journal of Mathematical Analysis and Applications, vol. 265, no. 2, pp. 229-248, 2002.

[21] D. Matignon, "Stability result on fractional differential equations with applications to control processing," in Proceedings of the IEEE-SMC Computational Engineering in Systems and Application Multiconference (IMACS '96), pp. 963-968, Lille, France, July 1996.

[22] E. Ahmed, A. M. A. El-Sayed, and H. A. A. El-Saka, "Equilibrium points, stability and numerical solutions of fractional-order predator-prey and rabies models," Journal of Mathematical Analysis and Applications, vol. 325, no. 1, pp. 542-553, 2007.

[23] Y. Chen, H.-S. Ahn, and I. Podlubny, "Robust stability check of fractional order linear time invariant systems with interval uncertainties," in Proceedings of the IEEE International Conference on Mechatronics and Automation (ICMA '05), pp. 210-215, Niagara Falls, Canada, July 2005.

[24] S. Boyd and C. Barratt, Linear Controller Design: Limits of Performance, chapter 1, Prentice-Hall, Upper Saddle River, NJ, USA, 1991.

[25] A. Arneodo, P. H. Coullet, E. A. Spiegel, and C. Tresser, "Asymptotic chaos," Physica D, vol. 14, no. 3, pp. 327-347, 1985.

[26] M. S. Tavazoei and M. Haeri, "A necessary condition for double scroll attractor existence in fractionalorder systems," Physics Letters A, vol. 367, no. 1-2, pp. 102-113, 2007.

[27] M.S. Tavazoei and M. Haeri, "Chaotic attractors in incommensurate fractional order systems," Physica D, vol. 237, no. 20, pp. 2628-2637, 2008. 